


\title{
DAS TIERREICH
}

\section{Eine Zusammenstellung und Kennzeichnung}

\author{
der rezenten Tierformen
}

The ,TIERREICH“" was initiated by the German Zoological Society in order to create a complete systematical treatment of all groups of the recent animal kingdom. After the World War II this undertaking was stopped because it seemed impossible to attain its completeness. In consequence of that, however, also ceased both incitement and possibility of publishing comprehensive revisions of large animal groups in a continued series. Therefore the Verlag Walter de Gruyter \& Cie. (Berlin) came to the conclusion to revive the ,TIERREICH". The original views will be maintained, the work, however, is planned now as an unconstrained sequence of systematical monographs without intending to treat all zoological categories according to a fixed plan. These monographs should not be restrieted only to the belongs of taxionomy but also have regard to anatomical, biological, physiological, and other facts as far as they are able to characterize the group in question without breaking the frame of the whole work. All authors who are willing to publish systematical revisions of recent animal groups are invited to collaborate. According to the wishes of the authors, their contributions will be printed in English, French, or German. The authors will get an honorarium of DM 55,- for each 16 printed pages and 5 free copies after come out of their contributions. Further details may be gathered from the exemplaries no. 72 and subsequents published since 1958; by request the publishers will give any informations or send exact directions for the authors. Summary revisions of zoological groups (checklists) will although be published in the series ,DAS TIERREICH“".

Le ,TIERREICH“ fut fondé pa la Société Allemande de Zoologie dans le but de réaliser une présentation complète de la systématique de tous les groupes zoologiques récents. Cette entreprise fut suspendue après la deuxième guerre mondiale car la présentation complète que l'on s'était fixée pour but semblait impossible à réaliser. Mais, de ce fait, disparut, avec la possibilité, le stimulant nécessaire à la publication continue d'amples révisions de groupes zoologiques importants. C'est pourquoi, les éditions Walter de Gruyter \& Co. (Berlin) ont pris la décision de faire renaître le ,TIERREICH“ sous l'optique originale pour ce qui est du fond, certes, mais uniquement sous la forme d'une série de monographies systématiques, sans lien étroit entre elles dont le but ne sera pas de traiter tous les groupes zoologiques d'après un plan préconçu. Ces monographies ne doivent pas simplement se borner au domaine de la taxionomie, mais tenir compte également des faits anatomiques, biologiques, physiologiques et autres dans la mesure où ils sont susceptibles de mieux caractériser le groupe 


\section{DAS TIERREICH}

Eine Zusammenstellung und Kennzeichnung der rezenten Tierformen

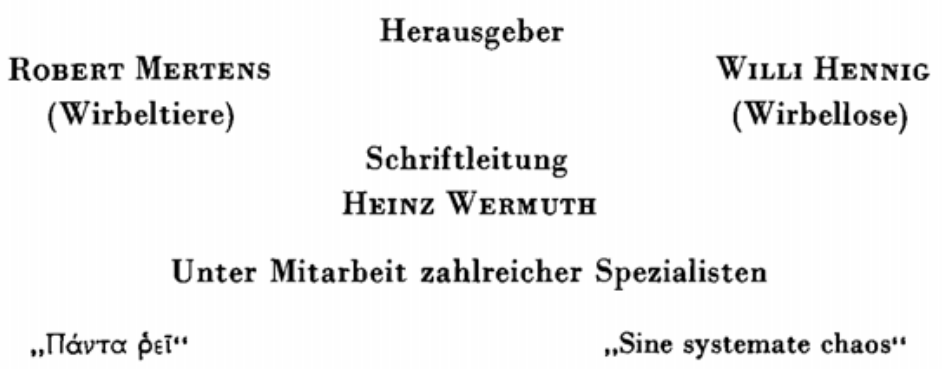

Lieferung 97

Lepidoptera Noctuiformes

Agaristidae I

(Palaearctic and Oriental Genera)

Bearbeitet von

Prof. Dr. S. G. Kiriakoff, Gent

Mit 39 Figuren im Text

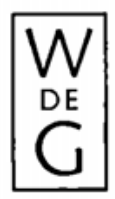

Walter de Gruyter • Berlin • New York

1977

\begin{tabular}{|l|l|l|l|l|}
\hline Das Tierreich & Berlin & Lieferung 97 & Seite I-IX, 1-180 & 1977 \\
\hline
\end{tabular}


ISBN 3110058766

(C) 1976 by Walter de Gruyter \& Co., vormals G. J. Göschen'sche Verlagshandlung, J. Guttentag, Verlagsbuchhandlung Georg Reimer, Karl J. Trübner, Veit \& Comp., Berlin 30.

Alle Rechte, insbesondere das Recht der Vervielfältigung und Verbreitung, sowie der Ubersetzung, vorbehalten. Kein Teil des Werkes darf in irgendeiner Form (durch Photokopic, Mikrofilm oder ein anderes Verfahren) ohne schriftliche Genehmigung des Verlages reproduziert oder unter Verwendung elektronischer Systeme verarbeitet, vervielfältigt oder verbreitet werden. Printed in Germany.

Satz: IBM-Composer Walter de Gruyter \& Co., Berlin.

Druck: Karl Gerike, Berlin.

Bindearbeiten: Dieter Mikolai, Berlin. 


\section{Familia Agaristidae I \\ (Palaearctic and Oriental Genera)}

\section{Bearbeitet von}

Prof. Dr. S. G. Kiriakoff 
